\title{
STARK ZEROS IN CERTAIN TOWERS OF FIELDS
}

\author{
V. Kumar Murty
}

Table of Contents

1. Introduction

2. Group theory

3. Zero-free regions for L-functions

4. Solvable Galois closure

5. Application to the Brauer-Siegel theorem

\section{Introduction}

It has long been known that zeros of the Dedekind zeta function near $s=1$ have a mitigating effect on the growth of the residue at $s=1$. In some cases, this can be translated into an effect on the growth of class numbers. All attempts thus far to prove that such zeros do not exist have been unsuccesful. However, in some applications, it suffices to know that the appearance of these zeros is a rare phenomenon. The main results of this paper consider a tower of fields, each member of which has solvable normal closure over its predecessor. In this case, we show that (in a sense to be made precise below), zeros near $s=1$ cannot persist as we move up the tower.

To state this precisely, we introduce some notation. Let $K$ be a number field and denote by $d_{K}$ the absolute value of the discriminant of $K / \mathbb{Q}$. Denote by $\rho_{K}$ the residue at $s=1$ of the Dedekind zeta function $\zeta_{K}(s)$ of $K$. It is a well-known result of Stark [10] that for $0<c<1 / 4, \zeta_{K}(s)$ has at most one zero in the region

$$
1-\frac{c}{\log d_{K}} \leq \sigma \leq 1, \quad|t| \leq \frac{c}{\log d_{K}} .
$$

This zero, if it exists, is necessarily real and simple. We shall call such a zero a Stark zero (or more precisely, a $c$-Stark zero) and denote it by $\beta=\beta_{K}$.

The concept of a Stark zero is similar to, but different from, that of a Siegel zero. Consider the cyclotomic field $K=\mathbb{Q}\left(\zeta_{q}\right)$. It is known that $\zeta_{K}(s)$ has at most one zero in the region

$$
1-\frac{c}{\log q} \leq \sigma \leq 1, \quad|t| \leq \frac{c}{\log q}
$$

Received July 15, 1998.

E.W.R. Steacie Fellow.

This paper was written while the author was visiting Harvard University. He would like to thank Barry Mazur for the invitation to visit and for many helpful and inspiring conversations, and the Department of Mathematics for its friendly working conditions. 
provided $c>0$ is sufficiently small. The best known value of $c$ (due to HeathBrown [5]) is $c=.348$. This exceptional zero, if it exists, is called a Siegel zero. Note that this region is much wider than the region in which a Stark zero can occur. Indeed, in this case,

$$
\log d_{K} \sim q \log q .
$$

For a general field, the concept of a Siegel zero should be defined in terms of Artin conductors. We give such a definition in $\S 3$.

Stark showed that if $L / K$ is a (finite) Galois extension and $\zeta_{L}(s)$ has a simple zero at $s=s_{0}$, then there is a field $F$ with

$$
K \subseteq F \subseteq L
$$

such that

(i) $F / K$ is cyclic,

(ii) $\zeta_{F}\left(s_{0}\right)=0$,

(iii) if $K \subseteq E \subseteq L$ and $\zeta_{E}\left(s_{0}\right)=0$, then $F \subseteq E$.

Moreover, if $s_{0} \in \mathbb{R}$, then $[F: K] \leq 2$. This paper is based on a generalization (Theorem 4.1) of Stark's theorem to certain non-normal extensions.

The problem of weakening the requirement that $L / K$ be normal in the theorem quoted above was also considered by Stark [10] himself. Denote by $\tilde{L}$ the normal closure of $L / K$. By the Aramata-Brauer theorem, $\zeta_{L}(s)$ divides $\zeta_{\tilde{L}}(s)$, in the sense that the quotient $\zeta_{\tilde{L}}(s) / \zeta_{L}(s)$ is entire. Thus, $\zeta_{\tilde{L}}\left(s_{0}\right)=0$. However, in general, it may no longer be a simple zero and so the above theorem of Stark no longer applies. To deal with this, Stark considered zeros sufficiently near $s=1$ where simplicity could be forced. In particular, this would be the case if $s_{0}$ were a Stark zero of $\zeta_{\tilde{L}}(s)$. Let $n=[K: \mathbb{Q}]$ and $g(n)=n$ !. If $s_{0}=\beta_{K}$ satisfies the stronger condition

$$
\beta \geq 1-\frac{1}{4 n ! \log d_{K}}
$$

then Stark showed that there is an extension $F$ with $\mathbb{Q} \subseteq F \subseteq K,[F: \mathbb{Q}] \leq 2$ and such that $\zeta_{F}(\beta)=0$.

By considering special classes of extensions, several authors have attempted to weaken the condition (4) in which this conclusion can be made. In particular, Odlyzko and Skinner [9] considered towers of radical extensions

$$
\mathbb{Q}=K_{0} \subseteq K_{1} \subseteq \cdots \subseteq K_{m}=K .
$$

Thus for each $i, K_{i+1}=K_{i}\left(\alpha_{i}^{1 / n_{i}}\right)$ for some $\alpha_{i} \in K_{i}$ and $1 \leq n_{i} \in \mathbb{Z}$. Assuming that

$$
\left[K_{i+1}: K_{i}\right] \text { is odd for all } i,
$$

they proved that $\zeta_{K}(s)$ has no Stark zeros. They asked whether a similar result could be proved if radical extensions were replaced with extensions having solvable normal closure. In $\S 4$, we shall prove such a result. Our method is actually simpler than [9]. 
The Brauer-Siegel theorem asserts that the residue $\rho_{K}$ of $\zeta_{K}(s)$ at $s=1$ satisfies

$$
\frac{\log \rho_{K}}{\log d_{K}} \longrightarrow 0
$$

as we range through a sequence of fields $K$ with

$$
\frac{1}{n_{K}} \log d_{K} \longrightarrow \infty
$$

Here, $n_{K}=[K: \mathbb{Q}]$. Thus, for example, if we range through all imaginary quadratic fields, then this says that

$$
\frac{\log h_{K} / \sqrt{d_{K}}}{\log d_{K}} \longrightarrow 0
$$

or what is the same,

$$
\frac{\log h_{K}}{\log d_{K}} \longrightarrow \frac{1}{2} \quad \text { as } d_{K} \longrightarrow \infty
$$

When one attempts to make this effective, difficulties are encountered because of possible zeros of $\zeta_{K}(s)$ near $s=1$. Though such zeros cannot be eliminated, Stark [10] used his result on simple zeros to show that they do not propagate in towers of fields, each element of which is normal over the predecessor. We prove (Proposition 5.1) an analogue of this for certain non-normal towers.

The relation between zeros near $s=1$ and $\rho_{K}$ has been made very explicit in Stark [10]. In particular, he shows that

$$
\rho_{K}>c_{1}\left(1-\beta_{1}\right),
$$

where $c_{1}>0$ is an absolute and effective constant and

$$
\beta_{1}= \begin{cases}\beta_{K} & \text { if it exists, } \\ 1-\frac{1}{4 \log d_{K}} & \text { otherwise }\end{cases}
$$

and $\beta_{K}$ is the possible zero in (1). Moreover, if $K / \mathbb{Q}$ is Galois, the existence of $F$ in (3) with $\zeta_{F}\left(\beta_{K}\right)=0$ implies that

$$
\beta_{K}<1-\frac{c_{2}}{d_{F}^{1 / 2}}
$$

for $c_{2}>0$ absolute and effective. Since

$$
d_{K} \geq d_{F}^{n_{K} / 2}
$$

this gives

$$
\rho_{K} \geq \frac{c_{2}}{d_{F}^{1 / 2}}>\frac{c_{2}}{d_{K}^{1 / n_{K}}}=c_{2} \exp \left(-\frac{1}{n_{K}} \log d_{K}\right)
$$

In $\S 5$, we note how the result of $\S 4$ can be similarly applied. 


\section{Group theory}

Let $F$ be a field and denote by $F^{s}$ a separable algebraic closure. Let $V$ be a vector space of dimension $r$ over $F$. A subgroup $G$ of $\mathrm{GL}(V) \simeq \mathrm{GL}_{r}(F)$ is irreducible if the only $F$-subspaces of $V$ invariant under $G$ are $\{0\}$ and $V$. We say that $G$ is completely reducible if $V=W_{1} \oplus \cdots \oplus W_{s}$, with each $W_{i}$ irreducible.

A subgroup $H$ of $G$ is called subnormal if there is a finite chain of subgroups

$$
G=H_{0} \supseteq H_{1} \supseteq \cdots \supseteq H_{k}=H,
$$

with $H_{i+1}$ normal in $H_{i}, i=0,1, \ldots, k-1$. We need the following result of Dixon ([2], Theorem 2).

Theorem 2.1. Let $G$ be a solvable, completely reducible subgroup of $\mathrm{GL}_{r}\left(F^{s}\right)$. Then $G$ has a subnormal Abelian subgroup $A$ with

$$
[G: A] \leq \gamma(r)
$$

where

$$
\gamma(r)=12^{r-1} 3^{1 / 3}
$$

Actually, we need the following small variant.

Proposition 2.1. Suppose $H$ is an irreducible, solvable subroup of $\mathrm{GL}_{r}\left(\mathbb{F}_{p}\right)$. Then $H$ has a subnormal Abelian subgroup of index $\leq \gamma(r)$.

Proof. (Dixon) As $H$ is irreducible, it does not contain any normal $p$-subgroup larger than $\{1\}$. Hence, by [3], Corollary $2.4, H$ is completely reducible, viewed as a subgroup of $\mathrm{GL}_{r}\left(\overline{\mathbb{F}}_{p}\right)$. Now the conclusion follows from Theorem 2.1.

\section{Zero-free regions for $L$-functions}

We begin by defining the concept of a Siegel zero of $\zeta_{K}(s)$ for any (finite) extension $K / \mathbb{Q}$. Let $\tilde{K} / \mathbb{Q}$ be the normal closure of $K / \mathbb{Q}$ and let $H$ denote $\operatorname{Gal}(\tilde{K} / K)$. Let $\chi$ be an irreducible character of $\operatorname{Gal}(\tilde{K} / \mathbb{Q})$ and denote by $\mathfrak{f}_{\chi}$ the corresponding Artin conductor. Set

$$
A=\max \left|\mathfrak{f}_{\chi}\right|,
$$

where the maximum ranges over those characters $\chi$ for which $\left(\left.\chi\right|_{H}, 1\right)>0$. We make the following plausible conjecture.

Conjecture 3.1. There exists $c>0$, absolute and effective, such that $\zeta_{K}(s)$ has at most one zero in the region

$$
1-\frac{c}{\log A} \leq \sigma \leq 1, \quad|t| \leq \frac{c}{\log A} .
$$

This zero, if it exists, is real and simple.

Definition 3.1. A zero in the region (14) is called a Siegel zero (or more precisely, a c-Siegel zero). 
This usage is then consistent with what is known to hold in the cyclotomic case as explained in the Introduction. Conjecture 3.1 is known to hold for $\tilde{K}=K$ Abelian over $\mathbb{Q}$.

To state what is known in general, we need some notation. Let $L / K$ be a Galois extension of number fields. For a character $\chi$ of $\operatorname{Gal}(L / K)$, let us set

$$
A_{\chi}=d_{K}^{\chi(1)} \mathrm{N}_{K / \mathbb{Q}} \mathfrak{f}_{\chi} \text {. }
$$

Definition 3.2. We shall say that Artin's conjecture holds for $L / K$ if the Artin $L$-function $L(s, \chi)$ is entire for each irreducible non-identity character $\chi$ of $\operatorname{Gal}(L / K)$.

Under the assumption of Artin's conjecture for $L / K$, one has the following result ([6], Proposition 3.8).

Proposition 3.1. Let $L / K$ be a Galois extension and assume that Artin's conjecture holds for $L / K$. Set

$$
A=\max A_{\chi}, \quad d=\max \chi(1) .
$$

where the maximum is taken over all irreducible characters $\chi$ of $\operatorname{Gal}(L / K)$. Then there is a constant $0<c_{1} \leq 1$, absolute and effective, such that $\zeta_{L}(s)$ has at most one zero in the region

$$
1-\frac{c_{1}}{d^{3} \log A} \leq \sigma \leq 1, \quad|t| \leq \frac{c_{1}}{d^{3} \log A} .
$$

If it exists, this zero, $\beta_{1}$ (say), is necessarily simple, real and belongs to a character $\chi_{1}$ (say) which is Abelian and real.

Remark. It would be of interest to eliminate the factor $d^{3}$ in the above.

A conjecture related to Conjecture 3.1 is the following.

Conjecture 3.2. There exists a constant $c>0$, absolute and effective, such that $\zeta_{K}(s)$ has at most one zero in the region

$$
1-\frac{c n_{K}}{\log d_{K}} \leq \sigma \leq 1, \quad|t| \leq \frac{c n_{K}}{\log d_{K}} .
$$

Next, we need some estimates for $A_{\chi}$ in the Abelian case.

Proposition 3.2. Let $L / K$ be an Abelian extension. Then

$$
\max _{\chi} \log A_{\chi} \leq \frac{2}{[L: K]} \log d_{L}
$$

where the maximum ranges over all irreducible characters of $\operatorname{Gal}(L / K)$.

Proof. First, consider the case of $L / K$ cyclic of prime power order. The maximum on the left is attained by a character $\psi$ which generates the group of characters. From this, we see that

$$
A_{\psi^{i}}=A_{\psi}
$$


if $\psi^{i}$ is also a generator. Let $t=[L: K]$. By the conductor-discriminant formula,

$$
A_{\psi}^{\phi(t)} \leq d_{L}
$$

If $t=p^{\alpha}$, this gives

$$
\log A_{\psi} \leq \frac{1}{p^{\alpha-1}(p-1)} \log d_{L}=\frac{1}{t}\left(1+\frac{1}{p-1}\right) \log d_{L} \leq \frac{2}{t} \log d_{L} .
$$

Second, consider the case of a general cyclic extension. Then, $L$ is the compositum of disjoint extensions $L_{1}, \ldots, L_{r}$ with $L_{i} / K$ cyclic of order $t_{i}=p_{i}^{\alpha_{i}}$, and $t=p_{1}^{\alpha_{1}} \cdots p_{r}^{\alpha_{r}}$. Moreover, we can write $\psi=\psi_{1} \cdots \psi_{r}$, with each $\psi_{i}$ a character of $\operatorname{Gal}\left(L_{i} / K\right)$. If $\psi$ is a generator, so is each $\psi_{i}$ a generator of the character group of $\operatorname{Gal}\left(L_{i} / K\right)$. Moreover, $A_{\psi}$ divides $A_{\psi_{1}} \cdots A_{\psi_{r}}$. Hence

$$
\log A_{\psi} \leq \sum \log A_{\psi_{i}} \leq \sum \frac{2}{t_{i}} \log d_{L_{i}}=\frac{2}{t} \log d_{L}
$$

Third, in the general case, let us say that

$$
\log A_{\psi}=\max _{\chi} \log A_{\chi}
$$

The fixed field of the kernel of $\psi$ gives a cyclic extension $L_{\psi}$ of $K$. Then by the previous case,

$$
\begin{aligned}
{[L: K] \log A_{\psi} } & \leq 2\left[L: L_{\psi}\right] \log d_{L_{\psi}} \\
& \leq 2 \log d_{L}
\end{aligned}
$$

\section{Solvable Galois extensions}

The main result of this section generalizes the result of Odlyzko-Skinner [9] to extensions with solvable normal closure.

We need some notation. Let

$$
n=[L: K]
$$

and

$$
e(n)=\max _{p^{\alpha} \| n} \alpha
$$

Then set

$$
\delta(n)=(e(n)+1)^{2} \gamma(e(n))=(e(n)+1)^{2} 3^{1 / 3} 12^{e(n)-1}
$$

Since

$$
e(n) \leq \frac{\log n}{\log 2}
$$

we certainly have

$$
\delta(n) \ll n^{4} .
$$

Let $c_{1}>0$ be as in Proposition 3.1. 
Theorem 4.1. Let $0<c<c_{1} / 2$ and set $n=[L: K]$. Suppose that $n$ is odd and that the Galois closure $\tilde{L}$ of $L / K$ is solvable over $K$. Suppose that $\zeta_{L}(s)$ has a zero $\beta$ in the range

$$
1-\frac{c}{\delta(n) \log d_{L}} \leq \sigma \leq 1, \quad|t| \leq \frac{c}{\delta(n) \log d_{L}}
$$

Then, $\zeta_{K}(\beta)=0$.

Remark. Note that this zero $\beta$ is necessarily real and simple because it lies in the Stark region (1).

Proof. Let $G=\operatorname{Gal}(\tilde{L} / K)$ and $H=\operatorname{Gal}(\tilde{L} / L)$. We shall proceed by induction on the degree $n=[L: K]$. As $\tilde{L}$ is the normal closure, $H$ does not contain any nontrivial normal subgroup of $G$. Assume first that $H$ is maximal. Let $A$ be a minimal normal subgroup. Then $G=H A$. Moreover, $H \cap A=\{1\}$. Indeed, $H \cap A$ is normalized by $H$ (as $H$ normalizes itself and $A$ is normal in $G$ ) and by $A$ (as $A$ is Abelian and so commutes with $H \cap A$ ). Thus, it is normal in $G$ and as it is contained in $H$, it must be trivial.

The action of $H$ on $A$ is given by a map

$$
H \longrightarrow \operatorname{Aut}(A) \text {. }
$$

This action is faithful, as the kernel is a normal subgroup of $G$ contained in $H$. As $G$ is solvable, $A$ is elementary Abelian, say $A \simeq(\mathbb{Z} / p)^{r}$. Notice that

$$
p^{r}=|A|=[L: K] .
$$

Thus we have an inclusion

$$
H \hookrightarrow \mathrm{GL}_{r}(\mathbb{Z} / p) .
$$

This identifies $H$ with an irreducible subgroup of $\mathrm{GL}_{r}(\mathbb{Z} / p)$, for any invariant subspace would be normal in $G$ and contained in $A$. Now, we can apply Proposition 2.1 to deduce that there is a subnormal Abelian subgroup $H_{1}$ of $H$ (say) with

$$
\left[H: H_{1}\right] \leq \gamma(r)
$$

Let $L_{1}$ denote the subfield of $\tilde{L}$ fixed by $H_{1}$. We have

$$
\left|H_{1}\right| \log d_{L_{1}} \leq \log d_{\tilde{L}}
$$

On the other hand, let $a_{1}, \cdots, a_{r}$ be generators of $A$. Then

$$
H \bigcap\left(\cap a_{i} H a_{i}^{-1}\right)=\{1\},
$$

as it is a normal subgroup of $G$ in $H$. Hence, $\tilde{L}$ is the compositum of $r+1$ conjugates of $L$. Hence,

$$
\log d_{\tilde{L}} \leq|H|(r+1) \log d_{L}
$$

Putting these estimates together, we deduce that

$$
\log d_{L_{1}} \leq(r+1) \gamma(r) \log d_{L} .
$$


Hence,

$$
1-\frac{c}{(r+1) \log d_{L_{1}}} \leq 1-\frac{c}{\delta(n) \log d_{L}}
$$

Now, as $H_{1}$ is subnormal in $H$, we can apply the Aramata-Brauer theorem in stages to deduce that $\zeta_{L}(s)$ divides $\zeta_{L_{1}}(s)$. In particular, $\zeta_{L_{1}}(s)$ vanishes at $s=\beta$. Moreover, as it lies in the Stark region (1), it is a simple zero.

Next, we wish to show that this is a simple zero of $\zeta_{\tilde{L}}(s)$. To do this, it suffices to show that it falls into the region considered in Proposition 3.1. Applying Proposition 3.2, we see that for any character $\chi$ of $\operatorname{Gal}\left(\tilde{L} / L_{1}\right)$,

$$
\log A_{\chi} \leq \frac{2}{\left[\tilde{L}: L_{1}\right]} \log d_{\tilde{L}} \leq \frac{2}{\left[\tilde{L}: L_{1}\right]}(r+1)|H| \log d_{L}
$$

and so

$$
\log A_{\chi} \leq 2(r+1) \log d_{L_{1}}
$$

Thus, $\beta$ is a simple real zero of $\zeta_{\tilde{L}}(s)$ and by Stark's theorem, there is an extension $N$ with

$$
K \subseteq N \subseteq \tilde{L},
$$

with $[N: K] \leq 2$ and $\zeta_{N}(\beta)=0$. Moreover, by the uniqueness part, and as $\zeta_{L}(\beta)=0$, it follows that

$$
K \subseteq N \subseteq L
$$

As $[L: K]$ is assumed to be odd, $N=K$.

Now consider the case when $H$ is not necessarily maximal. Suppose that

$$
H \subset H_{1} \subset G,
$$

and let $L_{1}$ denote the fixed field of $H_{1}$. Then $L / L_{1}$ has solvable normal closure. Moreover, if $m=\left[L: L_{1}\right]$, then $m$ is odd, $m<n$ and $\delta(m) \leq \delta(n)$ so the induction hypothesis applies and we can conclude that $\zeta_{L_{1}}(\beta)=0$. Now we apply the theorem to $L_{1} / K$ and the result follows by induction.

\section{Application to the Brauer-Siegel theorem}

We begin by proving a slight strengthening of Theorem 4.1. We will be considering the class $\mathfrak{S}$ of number fields $M$ which have solvable normal closure over $\mathbb{Q}$. We define a subclass $\mathfrak{S}_{R}$ of fields for which there is a chain

$$
\mathbb{Q}=M_{0} \subseteq M_{1} \subseteq \cdots \subseteq M_{t}=M,
$$

such that for each $1 \leq i \leq t$, the extension $M_{i} / M_{i-1}$ has odd degree and solvable normal closure and

$$
\max _{i} e\left(\left[M_{i+1}: M_{i}\right]\right) \leq R .
$$

Proposition 5.1. Let $M \in \mathfrak{S}_{R}$. Let $0<c<c_{1} / 2$. Then, $\zeta_{M}(s)$ does not have any real zeros in the range

$$
1-\frac{c}{(R+1)^{2} \gamma(R) \log d_{M}} \leq \sigma \leq 1
$$


Proof. Let $K \subseteq L \subseteq M$ be fields with $L / K$ and $M / L$ of odd degree and having solvable normal closure. Suppose that $\zeta_{M}(\beta)=0$ with $\beta$ in the range (16). By Theorem 4.1, $\zeta_{L}(\beta)=0$ Hence, we can shorten the sequence by one field and in doing this, we do not increase $R$. Moreover,

$$
\log d_{L} \leq \log d_{M}
$$

Hence, we can proceed inductively to deduce that $\zeta_{\mathbb{Q}}(\beta)=0$. But $\zeta_{\mathbb{Q}}$ has no real zeros in the critical strip. This proves the result.

Now using Proposition 5.1 and the estimate of Stark quoted in (7) we deduce the following.

Theorem 5.1. There is an effective absolute constant $c>0$ such that for any field $K$ in $\mathfrak{S}_{R}$, we have

$$
\rho_{K}>\frac{c}{(R+1)^{2} \gamma(R) \log d_{K}} .
$$

\section{References}

[1] H. Davenport, Multiplicative number theory, 2nd edition, Graduate Texts in Mathematics, 74, Springer-Verlag, New York-Berlin, 1980.

[2] J.D. Dixon, The Fitting subgroup of a linear solvable group, J. Austral. Math. Soc. 7 (1967), 417-424.

[3] , The structure of linear groups, Van Nostrand Reinhold, London, 1971.

[4] R. Foote and V. Kumar Murty, Zeros and poles of Artin L-series, Math. Proc. Cambridge Philos. Soc. 105 (1989), 5-11.

[5] R. Heath-Brown, Zero-free regions for Dirichlet L-functions, and the least prime in an arithmetic progression, Proc. London Math. Soc., 64 (1992), 265-338.

[6] V. Kumar Murty, Modular forms and the Chebotarev density theorem,II, in Analytic Number Theory, ed. Y. Motohashi, pp. 287-308, Cambridge University Press, Cambridge, 1997.

[7] _ Non-vanishing of L-functions and their derivatives, in Automorphic forms and analytic number theory, ed. R. Murty, pp. 89-113, Univ. Montreal, Montreal, 1990.

[8] M. Ram Murty and V. Kumar Murty, Non-vanishing of L-functions and applications, Progress in Mathematics, 157, Birkhauser Verlag, Basel, 1997.

[9] A. Odlyzko and C. Skinner, Non-existence of Siegel zeros in towers of radical extensions, Contemp. Math. 143 (1993), 499-511.

[10] H.M. Stark, Some effective cases of the Brauer-Siegel theorem, Invent. Math. 23 (1974), $135-152$.

Department of Mathematics, University of Toronto, 100 St. George Street, Toronto, ON M5S 3G3, CANADA

E-mail address: murty@math.toronto.edu 